\title{
ON THE BETTI NUMBERS OF REAL VARIETIES
}

\author{
J. MILNOR
}

The object of this note will be to give an upper bound for the sum of the Betti numbers of a real affine algebraic variety. (Added in proof. Similar results have been obtained by $\mathrm{R}$. Thom [10].)

Let $V$ be a variety in the real Cartesian space $R^{m}$, defined by polynomial equations

$$
f_{1}\left(x_{1}, \cdots, x_{m}\right)=0, \cdots, f_{p}\left(x_{1}, \cdots, x_{m}\right)=0 .
$$

The $q$ th Betti number of $V$ will mean the rank of the Cech cohomology group $H^{q}(V)$, using coefficients in some fixed field $F$.

THEOREM 2. If each polynomial $f_{i}$ has degree $\leqq k$, then the sum of the Betti numbers of $V$ is $\leqq k(2 k-1)^{m-1}$.

Analogous statements for complex and/or projective varieties will be given at the end.

I wish to thank W. May for suggesting this problem to me.

REMARK A. This is certainly not a best possible estimate. (Compare Remark B.) In the examples which I know, the sum of the Betti numbers of $V$ is always $\leqq k^{m}$. Consider for example the $m$ polynomials

$$
f_{i}\left(x_{1}, \cdots, x_{m}\right)=\left(x_{i}-1\right)\left(x_{i}-2\right) \cdots\left(x_{i}-k\right),
$$

where $i=1,2, \cdots, m$. These define a zero-dimensional variety, consisting of precisely $k^{m}$ points.

The proofs of Theorem 2 and of Theorem 1 (which will be stated later) depend on the following.

Lemma 1. Let $V_{0} \subset R^{m}$ be a zero-dimensional variety defined by polynomial equations $f_{1}=0, \cdots, f_{m}=0$. Suppose that the gradient vectors $d f_{1}, \cdots, d f_{m}$ are linearly independent at each point of $V_{0}$. Then the number of points in $V_{0}$ is at most equal to the product $\left(\operatorname{deg} f_{1}\right)\left(\operatorname{deg} f_{2}\right)$ -.. $\left(\operatorname{deg} f_{m}\right)$.

Proof. Approximate $f_{1}, \cdots, f_{m}$ by real polynomials $F_{1}, \cdots, F_{m}$ of the same degrees whose coefficients are algebraically independent. Now consider the variety $V_{C}$ in the complex Cartesian space $\boldsymbol{C}^{m}$ defined by the equations $F_{1}=0, \cdots, F_{m}=0$. It follows from van der Waerden $[9, \S 41]$ that the number of points in $V_{C}$ is equal to $\left(\operatorname{deg} f_{1}\right)\left(\operatorname{deg} f_{2}\right) \cdots\left(\operatorname{deg} f_{m}\right)$. Since each point of $V_{0}$ lies close to some real point of $V_{C}$; this proves Lemma 1.

Received by the editors January 17, 1963. 
Next let $W$ be a compact, nonsingular hypersurface in $\boldsymbol{R}^{m}$, defined by the equation $f=0$, where $f$ is a polynomial of degree $2 k$.

TheOREM 1. The sum of the Betti numbers of $W$ is $\leqq 2 k(2 k-1)^{m-1}$.

Similar estimates have been given by Olneĭnik [5].

The proof follows. Consider the function $n: W \rightarrow S^{m-1}$ which assigns to each point of $W$ its unit normal vector $\operatorname{grad} f /|\operatorname{grad} f|$. According to a theorem of Sard, the set of critical values of $\boldsymbol{n}$ has measure zero in $S^{m-1}$. (See [7] or [6, p. 10].) Hence, after rotating the coordinate system if necessary, we may assume that the two points $(0, \cdots, 0, \pm 1)$ of $S^{m-1}$ are not critical values of $n$. In terms of local coordinates $u_{1}, \cdots, u_{m-1}$ on $W$, this means that the matrix $\left(\partial n_{i} / \partial u_{j}\right)_{i, j<m}$ is nonsingular whenever $n=(0, \cdots, 0, \pm 1)$.

Now note that the "height function" $\left(x_{1}, \cdots, x_{m}\right) \rightarrow x_{m}$ on $W$ has no degenerate critical points. In fact near each critical point of this function we can choose local coordinates $u_{1}, \cdots, u_{m-1}$ so that

$$
x_{1}=u_{1}, \cdots, u_{m-1}=u_{m-1}, \quad x_{m}=h\left(u_{1}, \cdots, u_{m-1}\right),
$$

where $h$ is the height function. Then

$$
\begin{array}{r}
\mathbf{n}\left(u_{1}, \cdots, u_{m-1}\right) \\
\quad= \pm\left(\partial h / \partial u_{1}, \cdots, \partial h / \partial u_{m-1},-1\right) / \sqrt{ }\left(\sum\left(\partial h / \partial u_{j}\right)^{2}+1\right)
\end{array}
$$

hence

$$
\partial n_{i} / \partial u_{j}= \pm \partial^{2} h / \partial u_{i} \partial u_{j}
$$

at each critical point. Therefore the matrix $\left(\partial^{2} h / \partial u_{i} \partial u_{j}\right)$ is nonsingular; as was asserted.

Now applying Morse theory to the height function $h: W \rightarrow R$, it follows that the sum of the Betti numbers of $W$ is less than or equal to the number of critical points of $h$. (See for example Seifert and Threlfall $[8, \S \$ 5$ and 8$]$.

The critical points of $h$ can be characterized as the solutions of the $m$ polynomial equations

$$
\partial f / \partial x_{1}=0, \cdots, \partial f / \partial x_{m-1}=0, \quad f=0 .
$$

Note that at each critical point of $h$ the $m$ gradient vectors

$$
d\left(\partial f / \partial x_{1}\right), \cdots, d\left(\partial f / \partial x_{m-1}\right), d f
$$

are linearly independent.

Proof. If we differentiate the identity

$$
f\left(u_{1}, \cdots, u_{m-1}, h\left(u_{1}, \cdots, u_{m-1}\right)\right)=0
$$


twice, and then evaluate at a critical point, we obtain

$$
\partial^{2} f / \partial x_{i} \partial x_{j}+\left(\partial f / \partial x_{m}\right)\left(\partial^{2} h / \partial u_{i} \partial u_{j}\right)=0 ;
$$

which shows that the matrix $\left(\partial^{2} f / \partial x_{i} \partial x_{j}\right)_{i, j<m}$ is nonsingular. From this the linear independence of the $m$ gradient vectors follows easily.

Now Lemma 1 asserts that the number of critical points of $h$ is less than or equal to the product

$$
\left(\operatorname{deg} \partial f / \partial x_{1}\right) \cdots\left(\operatorname{deg} \partial f / \partial x_{m-1}\right)(\operatorname{deg} f)=(2 k-1)^{m-1} 2 k .
$$

Therefore the sum of the Betti numbers of $W$ is less than or equal to this number. This proves Theorem 1.

REMARK B. Using a more complicated argument, which applies Morse theory directly to the function $f$, one can prove the following slightly sharper inequality:

$$
\operatorname{rank} H^{*} W \leqq(2 k-1)^{m}+1 \text {. }
$$

Using this, Theorem 2 can be sharpened to the form

$$
\text { rank } H^{*} V \leqq \frac{1}{2}\left((2 k-1)^{m}+1\right) \text {. }
$$

Proof of Theorem 2. Let $r D^{m}$ denote the disk of radius $r$ in $R^{m}$. For each $r$ we will show that $H^{*}\left(V \cap r D^{m}\right)$ has rank $\leqq k(2 k-1)^{m-1}$.

Given numbers $\epsilon, \delta>0$ let $K(\epsilon, \delta)$ denote the set of points in $R^{m}$ for which

$$
f_{1}^{2}+\cdots+f_{p}^{2}+\epsilon^{2}\left(x_{1}^{2}+\cdots+x_{m}^{2}\right) \leqq \delta^{2} .
$$

This is a compact set, since it is contained in the disk of radius $\delta / \epsilon$. If $r \leqq \delta / \epsilon$ note that $V \cap r D^{m} \subset K(\epsilon, \delta)$. The set of points in $R^{m}$ for which the equality

$$
f_{1}^{2}+\cdots+f_{p}^{2}+\epsilon^{2}|x|^{2}=\delta^{2}
$$

holds will be denoted by $\partial K(\epsilon, \delta)$, and called the boundary of $K$. This is an algebraic hypersurface of degree $2 k$, where $k=\operatorname{Max}\left\{\operatorname{deg} f_{1}, \cdots\right.$, $\left.\operatorname{deg} f_{p}\right\} \geqq 1$. This hypersurface is nonsingular if and only if $\delta^{2}$ is a regular value of the function $f_{1}^{2}+\cdots+f_{p}^{2}+\epsilon^{2}|x|^{2}$. Hence, keeping $\epsilon$ fixed, it follows from Sard's theorem that this hypersurface is nonsingular for almost all values of $\delta$. We will assume henceforth that $\epsilon$ and $\delta$ are chosen so that this is the case.

Applying Theorem 1 to $\partial K$ we see that $H^{*}(\partial K)$ has rank $\leqq 2 k(2 k-1)^{m-1}$. But the Alexander duality theorem implies that

$$
\operatorname{rank} H^{*}(K)=\frac{1}{2} \operatorname{rank} H^{*}(\partial K) .
$$


(See for example [3, p. 279].) Hence

$$
\text { rank } H^{*} K \leqq k(2 k-1)^{m-1} \text {. }
$$

Now choose sequences $\left\{\epsilon_{i}\right\}$ and $\left\{\delta_{i}\right\}$ so that $\left\{\epsilon_{i}\right\}$ decreases monotonely with limit zero; so that $\left\{\delta_{i} / \epsilon_{i}\right\}$ decreases monotonely with limit $r$; and so that each $\partial K\left(\epsilon_{i}, \delta_{i}\right)$ is nonsingular. Then

$$
K\left(\epsilon_{1}, \delta_{1}\right) \supset K\left(\epsilon_{2}, \delta_{2}\right) \supset K\left(\epsilon_{3}, \delta_{3}\right) \supset \cdots
$$

with intersection $V \cap r D^{m}$. (These inclusion relations become clear if we put the defining inequality for $K(\epsilon, \delta)$ in the form

$$
\sum f_{j}^{2} / \delta^{2}+|x|^{2} \epsilon^{2} / \delta^{2} \leqq 1
$$

and note that $\delta_{i+1} \leqq \delta_{i} \epsilon_{i+1} / \epsilon_{i} \leqq \delta_{i}$.) Therefore the Čech cohomology group $H^{*}\left(V \cap r D^{m}\right)$ is equal to the direct limit of the groups $H^{*} K\left(\epsilon_{i}, \delta_{i}\right)$. (Compare Eilenberg and Steenrod [1, p. 261].) Hence

$$
\operatorname{rank} H^{*}\left(V \cap r D^{m}\right) \leqq \lim \sup \left(\operatorname{rank} H^{*} K\left(\epsilon_{i}, \delta_{i}\right)\right) \leqq k(2 k-1)^{m-1} .
$$

There remains one small but tricky point: the passage to the limit as $r \rightarrow \infty$. One approach is to make use of the theorem that $V$ can be triangulated. (See for example Lefschetz and Whitehead [2].) It follows that the Betti numbers of $V$ based on singular homology theory are the same as those based on Cech cohomology theory. But the singular homology group $H_{*}(V)$ is equal to the direct limit of the singular homology groups of the compact subsets ${ }^{1}$ of $V$. If $P \subset V \cap r D^{m}$ $C V$ is any compact sub-polyhedron, then the restriction homomorphism $H^{*} V \rightarrow H^{*} P$ has rank $\leqq k(2 k-1)^{m-1}$; hence the corresponding homology homomorphism $H_{*} P \rightarrow H_{*} V$ also has rank $\leqq k(2 k-1)^{m-1}$. Therefore the limit $H_{*} V$ has rank $\leqq k(2 k-1)^{m-1}$; which completes the proof.

An alternative approach which does not assume triangulability can be sketched as follows. By an argument similar to that in $[4$, p. 338] one constructs an exact sequence

$$
0 \rightarrow \mathfrak{L}^{\prime} H^{q-1}\left(V \cap r D^{m}\right) \rightarrow H^{q} V \rightarrow \mathcal{L} H^{q}\left(V \cap r D^{m}\right) \rightarrow 0,
$$

where $\mathscr{L}$ denotes the inverse limit functor, and $\mathscr{L}^{\prime}$ denotes its first derived functor. Since each $H^{q-1}\left(V \cap r D^{m}\right)$ is a finite-dimensional

\footnotetext{
${ }^{1}$ In contrast note that the Čech homology group of a space need not be the direct limit of the Cech homology groups of its compact subsets. A counter-example is provided by the union of the curve $y=\sin (1 / x) / x$ and the $y$-axis.
} 
vector space, an argument similar to that used by Eilenberg and Steenrod in $\left[1\right.$, p. 226] shows that the $\mathscr{L}^{\prime}$ term is zero. Therefore

$$
\operatorname{rank} H^{*} V=\operatorname{rank}\left(\mathscr{L} H^{*}\left(V \cap r D^{m}\right)\right) \leqq k(2 k-1)^{m-1},
$$

which completes the alternative proof.

As corollaries one obtains similar estimates, presumably rather crude, for complex or projective varieties.

Corollary 1 (The Complex AfFine CASE). If $V_{1} \subset C^{m}$ is defined by polynomial equations of degree $\leqq k$, then rank $H^{*} V_{1} \leqq k(2 k-1)^{2 m-1}$.

Corollary 2 (the complex projective case). If $V_{2} \subset P_{m}(C)$ is defined by homogeneous polynomial equations of degree $\leqq k$, then rank $H^{*} V_{2} \leqq m k(2 k-1)^{2 m+1}$.

Corollary 3 (the real projective Case). If $V_{3} \subset P_{m}(R)$ is defined by homogeneous polynomial equations of degree $\leqq k$, then rank $H^{*} V_{3}$ $\leqq m k(2 k-1)^{m}$. Here the initial factor $m$ in this expression is needed only in the special case when the coefficient field $F$ has characteristic 2.

In order to prove Corollary 1 it is only necessary to think of $V_{1}$ as a real variety in $R^{2 m}$. To prove Corollary 2 note that the same homogeneous equations can be used to define an affine variety $V_{2}^{\prime} \subset C^{m+1}$. The intersection $V_{2}^{\prime} \cap S^{2 m+1}$ is the total space of a circle bundle over $V_{2}$. Using the Gysin sequence of this bundle, one arrives at the given estimate. Corollary 3 is proved in the same way. In this case $V_{3}^{\prime} \cap S^{m}$ is a 2 -fold covering of $V_{3}$; so that the prime 2 plays a special role.

Added in proof. A rather different problem arises when one considers a locus which is defined by polynomial inequalities. In this case any estimate must depend not only on the degrees of the inequalities but also upon their number. As an example the $p$ quadratic inequalities

$$
(x-1)^{2} \geqq \frac{1}{10}, \quad(x-2)^{2} \geqq \frac{1}{10}, \cdots,(x-p)^{2} \geqq \frac{1}{10}
$$

on the real line define a locus with $p+1$ components.

Theorem 3. If $X \subset R^{m}$ is defined by polynomial inequalities of the form

$$
f_{1} \geqq 0, \cdots, f_{p} \geqq 0
$$

with total degree $d=\operatorname{deg} f_{1}+\cdots+\operatorname{deg} f_{p}$, then

$$
\operatorname{rank} H^{*} X \leqq \frac{1}{2}(2+d)(1+d)^{m-1} \text {. }
$$


Proof. Again we first replace $X$ by $X \cap r D^{m}$. This can be done by simply adjoining the additional inequality

$$
f_{0}=r^{2}-|x|^{2} \geqq 0 \text {. }
$$

Given numbers $\epsilon \geqq \epsilon^{p+1} \geqq \delta>0$, let $L(\epsilon, \delta)$ denote the set of points in for which

$$
f_{0}+\epsilon \geqq 0, \cdots, f_{p}+\epsilon \geqq 0
$$

and

$$
\left(f_{0}+\epsilon\right)\left(f_{1}+\epsilon\right) \cdots\left(f_{p}+\epsilon\right)-\delta \geqq 0 .
$$

This set is compact. Its boundary is obtained by setting only the last expression equal to zero.

Given $\epsilon$ we may assume that $\delta$ is chosen so that the boundary is nonsingular. Therefore the proof (if not the exact statement) of Theorem 1 shows that

$$
\text { rank } H^{*}(\partial L(\epsilon, \delta)) \leqq(2+d)(1+d)^{m-1} ;
$$

and hence that

$$
\operatorname{rank} H^{*} L(\epsilon, \delta) \leqq \frac{1}{2}(2+d)(1+d)^{m-1} .
$$

Now choose numbers $\epsilon_{i}$ tending monotonely to zero and choose $0<\delta_{i}<\left(\epsilon_{i}-\epsilon_{i+1}\right)^{p+1}$; so that $L\left(\epsilon_{1}, \delta_{1}\right) \supset L\left(\epsilon_{2}, \delta_{2}\right) \supset \ldots$ with intersection $X \cap r D^{m}$. As in the proof of Theorem 2, it follows that

rank $B^{*} X \leqq \lim \sup \left(\operatorname{rank} H^{*}\left(X \cap r D^{m}\right)\right) \leqq \frac{1}{2}(2+d)(1+d)^{m-1}$.

\section{REFERENCES}

1 S. Eilenberg and N. Steenrod, Foundations of algebraic topology, Princeton Univ. Press, Princeton, N. J., 1952.

2. S. Lefschetz and J. H. C. Whitehead, On analytical complexes, Trans. Amer. Math. Soc. 35 (1933), 510-517.

3. J. Milnor, On the immersion of $n$-manifolds in $(n+1)$-space, Comment. Math. Helv. 30 (1956), 275-284.

4. - On axiomatic homology theory, Pacific J. Math. 12 (1962), 337-341.

5. O. A. Oleynik, Estimates of the Betti numbers of real algebraic hypersurfaces, Mat. Sb. (N.S.) 28 (70) (1951), 635-640. (Russian)

6. G. de Rham, Variétes différentiables, Hermann, Paris, 1955.

7. A. Sard, The measure of the critical values of differentiable maps, Bull. Amer. Math. Soc. 48 (1942), 883-897.

8. H. Seifert and W. Threlfall, Variationstechnung im Grossen, Teubner, Berlin, 1938.

9. B. van der Waerden, Einführung in die algebraische Geometrie, Spinger, Berlin, 1939.

10. R. Thom, Sur l'homologie des variettés algébriques réelles, (to appear).

Princeton University 